\title{
Host-guest complexes of phenoxy alkyl acid herbicides and cyclodextrins. MCPA and $\beta$-cyclodextrin
}

\author{
E. MANUELA GARRIDO, MARCOS SANTOS, PAULO SILVA, FERNANDO CAGIDE, JORGE \\ GARRIDO and FERNANDA BORGES
}

\begin{abstract}
The chlorophenoxy herbicide MCPA (4-chloro-2-methylphenoxyacetic acid), widely used for the control of broad-leaf weeds primarily in cereal and grass seed crops, still remains one of the most often used herbicides in Portugal. As the formation of inclusion complexes with cyclodextrins can improve its solubility properties, the interaction between the herbicide MCPA and $\beta$-cyclodextrin was investigated. The stability constants describing the extent of formation of the complexes have been determined by phase-solubility studies. Different analytical techniques [ultraviolet-visible spectroscopy (UV-Vis), differential scanning calorimetry (DSC), Fourier transform infrared spectroscopy (FTIR), proton nuclear magnetic resonance spectroscopy $\left({ }^{1} \mathrm{H}\right.$ NMR)] were employed for a thorough investigation of the structural characteristics of the obtained complexes, which exhibited distinct features and properties from both "guest" and "host" molecules. FTIR and ${ }^{1} \mathrm{H}$ NMR data obtained for the MCPA/ $\beta$-CD complexes gave information about the interaction between MCPA and the nonpolar cyclodextrin cavity. The dramatic change observed in band frequency and proton displacements of $\mathrm{OCH}_{2}$ group and $\mathrm{H} 6$ aromatic proton confirmed the inclusion of MCPA in $\beta$-CD.

The formation of an inclusion complex between MCPA and $\beta$-CD increased the aqueous solubility of this herbicide which could be a particularly advantageous property for some specific applications, namely to improve commercial formulation and for environmental protection.
\end{abstract}

Keywords: Phenoxyacetic acid herbicides, MCPA, $\beta$-cyclodextrin, inclusion complex.

\section{Introduction}

As plants constitute the world's primary food source there has been a tendency, in recent years, to maximize agricultural yields due to demographical growth. The increase in productivity has been achieved through the development of new high-yield crops and the use of fertilizers and pesticides. At the global level, the annual losses due to the presence of destructive weeds are calculated to be around $10-15 \%$ of production among staple crops. Therefore, it is not outlandish that $47.5 \%$ of the 2 million tons of pesticides consumed globally each year are herbicides. ${ }^{[1]}$

The risk of environment contamination by pesticides and their degradation products has encouraged the interest in the development of new formulations for pesticide application. Slow-release formulations of pesticides maintain the threshold concentration of the active ingredient in the soil or plant by its release at the required rate, reducing its level in the environment because lower amounts are required for biological effect. These novel delivery approaches have been recently ascribed to encompass either economical or environmental advantages. ${ }^{[2-4]}$

Microencapsulation is a simple and cost-effective practice that allows enclosing bioactive materials with the purpose of protecting and/or releasing the enclosed substances in a controlled way under specific conditions. The encapsulation process involves coating or entrapment of a material or a mixture into another material.

Current encapsulation techniques included a variety of techniques, namely spray drying, spray chilling and cooling, coacervation, fluidized bed coating, liposome entrapment, rotational suspension separation, extrusion and inclusion complexation. The last method is generally achieved by using cyclodextrins (CDs) as the encapsulating material. The most important structural feature of these compounds is their toroid shape, with lipophilic inner cavities and hydrophilic outer surfaces, which make them capable of interacting with a large variety of guest molecules to form noncovalent inclusion complexes. ${ }^{[5,6]}$ Among them, $\beta$-CD is receiving increasing attention due its low cost and high capacity to interact with a wide variety of molecules, including pesticides and drugs. In fact, several studies have 
shown that synthetic pesticides can form inclusion complexes with CDs, often resulting in improvements in their chemical and physical properties, such as enhancement of solubility and bioavailability. ${ }^{[4,7-9]}$

The discovery of the phenoxyacetic acid herbicides during the Second World War heralded the modern era of agrochemicals. The fact that they are still being used today is proof of their efficacy, safety and low cost. These compounds work by imitating the natural plant growth hormone indole-3-acetic acid which causes abnormal growth that in turn results in plant death.

The chlorophenoxy herbicide MCPA (4-chloro-2methylphenoxyacetic acid) is widely used for the control of broad-leaf weeds primarily in cereal and grass seed crops. ${ }^{[10-12]}$ In Portugal, MCPA still remain one of the most often used herbicides for rice crops. ${ }^{[13]}$ MCPA is not extensively metabolized and urine is the predominant route of excretion. ${ }^{[14]}$ Studies conducted with whole animals indicate that MCPA is somewhat hazardous, although toxicological mechanisms have not yet been identified. ${ }^{[15]}$

As part of an extended project aimed to validate the application of nanotechnology for pesticide management, a complete study of the inclusion effect of CDs on the properties of MCPA was performed. Accordingly, inclusion complexes of MCPA with the host $\beta$-CD were synthesized by classical procedures often used in this research area. The stoichiometric ratios and stability constants describing the extent of formation of the complexes were obtained by phase-solubility studies. In addition, their structural characterization was done using different analytical tools such as ultraviolet-visible spectroscopy (UV-Vis), differential scanning calorimetry (DSC), Fourier transform infrared spectroscopy (FTIR) and proton nuclear magnetic resonance spectroscopy $\left({ }^{1} \mathrm{H}\right.$ NMR $)$.

\section{Materials and methods}

\section{Chemicals}

MCPA and $\beta$-cyclodextrin were purchased from SigmaAldrich Química S.A. (Sintra, Portugal). Deuterated solvents and tetramethylsilane (TMS) were obtained from Merck (Lisbon, Portugal). All other reagents and solvents were pro analysis grade and used without additional purification. Deionised water (conductivity $<0.1 \mu \mathrm{S} \mathrm{cm}^{-1}$ ) was used throughout all the experiments.

\section{Phase solubility studies}

Phase solubility studies were carried out according to the method described by Higuchi and Connors. ${ }^{[16]}$ Excess amount of MCPA (50 mg) was added to $25 \mathrm{~mL}$ of aqueous solutions containing various concentrations of $\beta$-CD $(0-0.01 \mathrm{M})$. Then, the suspensions were shaken on a incu- bator shaker at $25 \pm 2^{\circ} \mathrm{C}$ for 4 days. After equilibrium was reached, suspensions were centrifuged and the supernatant was withdrawn and properly diluted. The concentration of MCPA was determined spectrophotometrically (Shimadzu UV-Vis Spectrophotometer, UV-1700 PharmaSpec, Japan) at $228 \mathrm{~nm}$. The apparent stability constant, $K \mathrm{~s}$, was calculated from phase solubility diagrams with the assumption of 1:1 stoichiometry, according to Equation 1.

$$
K_{\mathrm{s}}=\text { slope } / \mathrm{S}_{0}(1-\text { slope })
$$

$S_{0}$ is the solubility of MCPA in the absence of CD.

\section{Preparation of the complexes}

The preparation of MCPA/ $\beta$-CD binary systems was performed by physical mixing, kneading and co-evaporation procedures.

\section{Physical mixtures (PM)}

Physical mixtures were prepared by simple mixing in a mortar, during 15 min., of previously weighted MCPA and $\beta$-CD.

\section{Kneading method (KN)}

MCPA and $\beta$-CD were accurately weighed and mixed together in different stoichiometric ratios $(1: 1,2: 1,3: 1$ and 1:2) using a mortar. Each mixture was then triturated and an appropriate amount of ethanol was added until a homogenous paste was formed. The paste was then kneaded for $45 \mathrm{~min}$ and dried at $45^{\circ} \mathrm{C}$ in an oven. The dried product was ground powdered and sieved through sieve no. 60 .

\section{Co-evaporation method (CE)}

MCPA/ $\beta$-CD complex in a 1:1 molar ratio was also prepared using the co-evaporation method. MCPA and $\beta$-CD (equimolar ratio) were completely dissolved in a solution of ethanol and water $(\mathrm{v} / \mathrm{v}=1: 20)$. The dispersion of MCPA in the aqueous $\beta$-CD solution was protected from light and mechanically shaken at room temperature and $100 \mathrm{rpm}$ in an IKA KS 4000i incubator shaker (IKA, Germany) for $48 \mathrm{~h}$ to achieve equilibrium of the complexation reaction. After evaporation of the ethanol from the reaction mixture, the uncomplexed MCPA was removed by filtration. The filtrate was evaporated under reduced pressure in a Büchi Rotavapor (Büchi, Germany) to remove the solvent and dried in vacuum to give the MCPA/ $\beta$-CD complex.

\section{Physicochemical characterization of MCPA / $\beta-C D$ complexes}

$U V$-visible spectroscopy. Spectrophotometric measurements were performed to quantify MCPA in its free and 
CD-complexed form. Standard curves of MCPA were prepared in deionised water.

Spectrophotometric scans were performed between 190 and $300 \mathrm{~nm}$ to monitor the UV spectra of MCPA. The absorbance maximum of $228 \mathrm{~nm}$ was used to quantify MCPA concentration.

Infrared spectroscopy. The FTIR spectra of MCPA, $\beta$-CD, the physical mixture and the inclusion complexes were recorded at room temperature on a Bruker FTIR Tensor 27 Spectrometer (Bruker, Billerica, MA) in a spectral region between 4000 and $400 \mathrm{~cm}^{-1}$. Samples were prepared as small pellets by mixing each of them in a mortar with $\mathrm{KBr}$ (1:100) followed by a hydraulic pressing (14 tons) operation.

Thermal analysis. DSC analysis was carried out for MCPA, $\beta$-CD, the physical mixture and inclusion complexes with a Netzsch DSC 204 calorimeter (Netzsch, Germany). The temperature scale was calibrated using In, Bi, Sn, $\mathrm{Zn}$ and $\mathrm{KNO}_{3}$ and the enthalpy calibration has been carried out to the heat of fusion of the same standards. Samples (2.5 to $3.0 \mathrm{mg}$ ) were placed in standard aluminum pans and measurements were performed at a heating rate of $10^{\circ} \mathrm{C} / \mathrm{min}$ from room temperature to $400^{\circ} \mathrm{C}$ in a dynamic nitrogen atmosphere (flow rate $=70 \mathrm{~mL} \mathrm{~min}^{-1}$ ). Reproducibility was checked by running the sample in triplicate.

\section{Nuclear magnetic resonance studies}

${ }^{1} \mathrm{H}$ NMR data were acquired, at room temperature, on a Bruker AMX 300 spectrometer operating at $300.13 \mathrm{MHz}$. Chemical shifts are expressed in $\delta(\mathrm{ppm})$ values relative to tetramethylsilane (TMS) as internal reference. Chemical shifts changes $(\Delta \delta)$ were calculated according to the formula $\Delta \delta=\delta_{\text {(complex) }}-\delta_{\text {(free) }}$.

All NMR experiments were carried out in deuterated water $\left(D_{2} \mathrm{O}\right)$, except for MCPA that was performed in deuterated methanol $\left(\mathrm{CD}_{3} \mathrm{OD}\right)$. Owing to the extremely poor aqueous solubility of MCPA, $\mathrm{D}_{2} \mathrm{O}$ was not used as a solvent. Nevertheless, the chemical shifts characteristic of MCPA molecule obtained in $\mathrm{CD}_{3} \mathrm{OD}$ are quite similar to that found in literature using $\mathrm{D}_{2} \mathrm{O}^{[17]}$

\section{Results and discussion}

\section{Phase solubility studies}

The stoichiometric ratio and stability constants were derived from the changes in the solubility of MCPA in the presence of increasing amounts of $\beta$-cyclodextrin, measured by UV spectrophotometry. Figure 1 presents the phase solubility plot obtained for MCPA and increasing concentrations of $\beta$-cyclodextrin in water at $25^{\circ} \mathrm{C}$. As can be seen, it shows an AL type solubility diagram as the MCPA solubility increases linearly with increasing $\beta$-CD

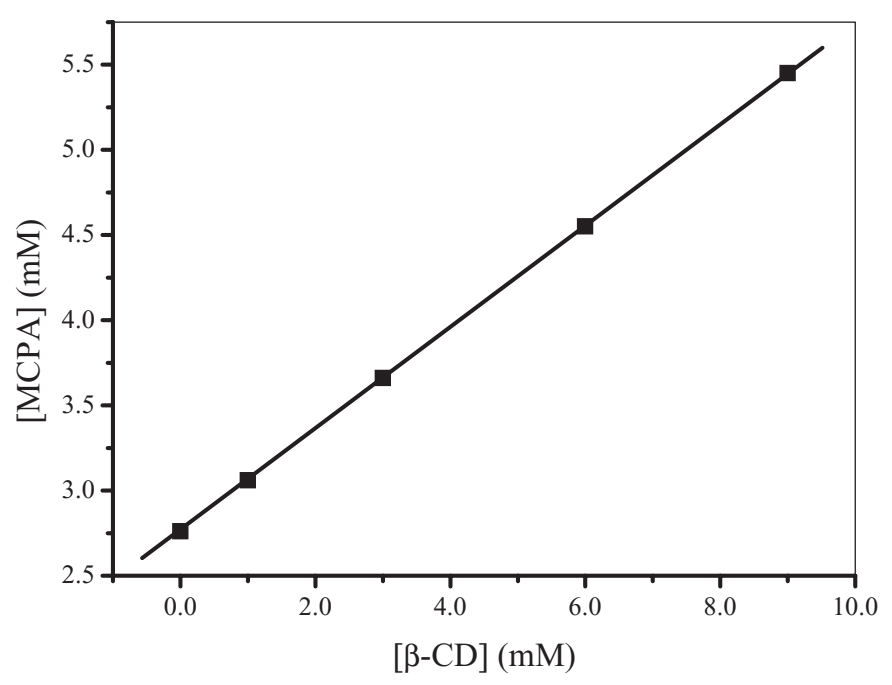

Fig. 1. Phase solubility diagram obtained for MCPA and increasing concentrations of $\beta$-cyclodextrin in water at $25^{\circ} \mathrm{C}$. Each point represents the mean of three determinations.

concentrations, according to the classification established by Higuchi and Connors. ${ }^{[16]}$ The diagram obtained indicates that MCPA solubility is apparently increased by the presence of the macrocycle. Since the slope of the diagram is less than 1, the complex stoichiometry was assumed to be $1: 1$. The stability constant, $K s$, was therefore calculated from the straight-line portion of the phase-solubility diagram, according to the equation previously presented. Table 1 summarizes data obtained from the phase solubility diagram (MCPA solubility, slope, stability constant and correlation coefficient).

\section{MCPA / $\beta-C D$ complexes}

Various methods have been used and widely reported for the formation of the cyclodextrin inclusion complexes. The selection of the method and its effectiveness highly depends on the nature of the drug and/or cyclodextrin. ${ }^{[18-20]}$ In this work the preparation of MCPA/ $\beta$-CD solid systems was performed using different techniques, namely by kneading and co-evaporation (see experimental part). To perform comparative evaluations, with the respective solid inclusion complexes, physical mixtures were also prepared.

\section{Physicochemical characterisation of the complexes}

Differential scanning calorimetry (DSC). DSC is an analytical technique often used for the recognition of inclusion complexes. When guest molecules are embedded into $\beta$ CD cavities, their melting, boiling or sublimating points generally shift to a different temperature or disappear. In accordance, the DSC profiles of pure components (MCPA, $\beta$-CD) and binary systems were acquired and the results obtained are shown in Figure 2. The DSC curve of $\beta$-CD 
Table 1. Complex formation parameters for MCPA $/ \beta$-CD system in water at $25^{\circ} \mathrm{C}$.

\begin{tabular}{lccc}
\hline$S_{0}(M)$ & $\alpha$ & $K s\left(M^{-1}\right)$ & $R^{2}$ \\
\hline 0.00276 & 0.299 & 154.5 & 0.9995 \\
\hline
\end{tabular}

$\mathrm{S}_{0}$ : represents the solubility of MCPA; $\alpha$ : slope, Ks: stability constant.

(Fig. 2A) shows a broad endothermic signal around $78^{\circ} \mathrm{C}$ that could be associated to the loss by evaporation of the water molecules existing as residual humidity. The broad endothermic effect occurring at $311.9^{\circ} \mathrm{C}$ is related to the melting of $\beta$-CD. The thermal curve of MCPA (Fig. 2A) was typical of a crystalline anhydrous substance with a sharp fusion endotherm at $119.8^{\circ} \mathrm{C}$, corresponding to the melting point of the compound, followed by an exothermal effect at $242.7^{\circ} \mathrm{C}$, attributed to its thermal decomposition. ${ }^{[21]}$

In the physical mixture (PM) DSC curves it was possible to clearly distinguish the MCPA fusion endothermic peak around $119.8^{\circ} \mathrm{C}$ in addition to the $\beta$-CD dehydra-

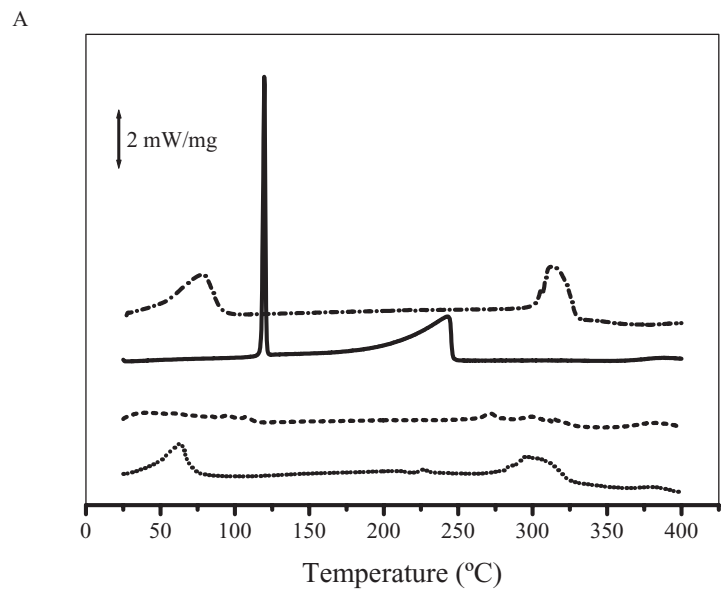

B

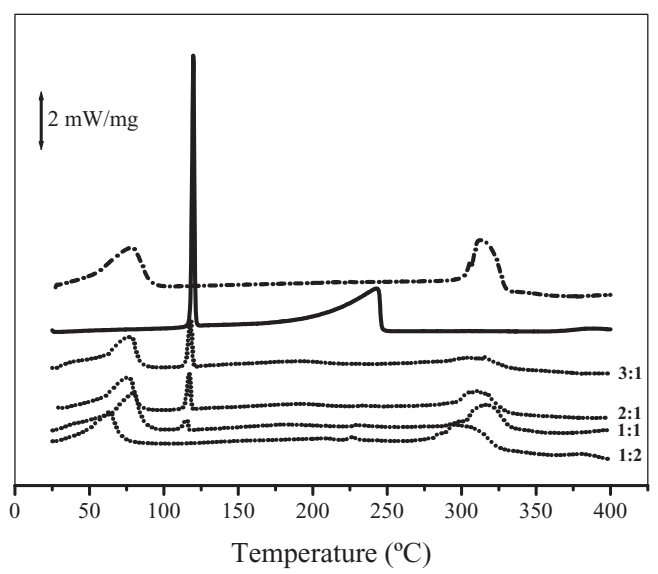

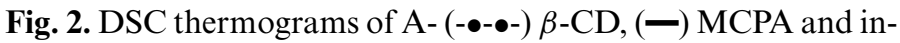

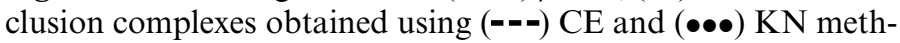

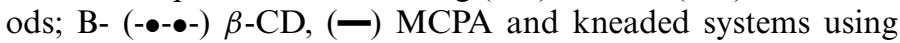
different MCPA/ $\beta$-CD stoichiometric ratios $(3: 1,2: 1,1: 1$ and $1: 2)$. tion process (data not shown). The obtained thermograms were in fact a superimposition of the individual components showing the absence of an interaction or a low yield of complexation between the herbicide and $\beta$-CD.

The thermal curve obtained for the solid inclusion complex of MCPA with $\beta$-CD obtained by co-evaporation method (CE) does not show the MCPA endothermic peak at $119.8^{\circ} \mathrm{C}$ (Fig. 2A). Its absence could be explained in view of the formation of a complex between herbicide and $\beta$-CD.

Considering KN systems, the absence of the MCPA melting endothermic peak was only noticed for MCPA/ $\beta$-CD stoichiometric ratio of 1:2 (Fig. 2B). Then, it is reasonable to assume that this method yield a 1:2 MCPA/ $\beta$-CD complex, because this stoichiometry would allow maximum contact between the hydrophobic portion of the organic substrate with the apolar cavity of $\beta$-CD.

The feasibility of formation of MCPA/ $\beta-\mathrm{CD}$ inclusion complexes using $\mathrm{CE}$ and $\mathrm{KN}$ methods were also confirmed by using other analytical techniques.

Infrared spectroscopy (FTIR). FTIR spectroscopy is a technique that could provide information about the chemical bonds and molecular structure of the "guest" molecules. Figure 3 presents the infrared spectra of MCPA, $\beta$ cyclodextrin and inclusion complexes, obtained using $\mathrm{CE}$ and $\mathrm{KN}$ methods, in the most significant spectral region.

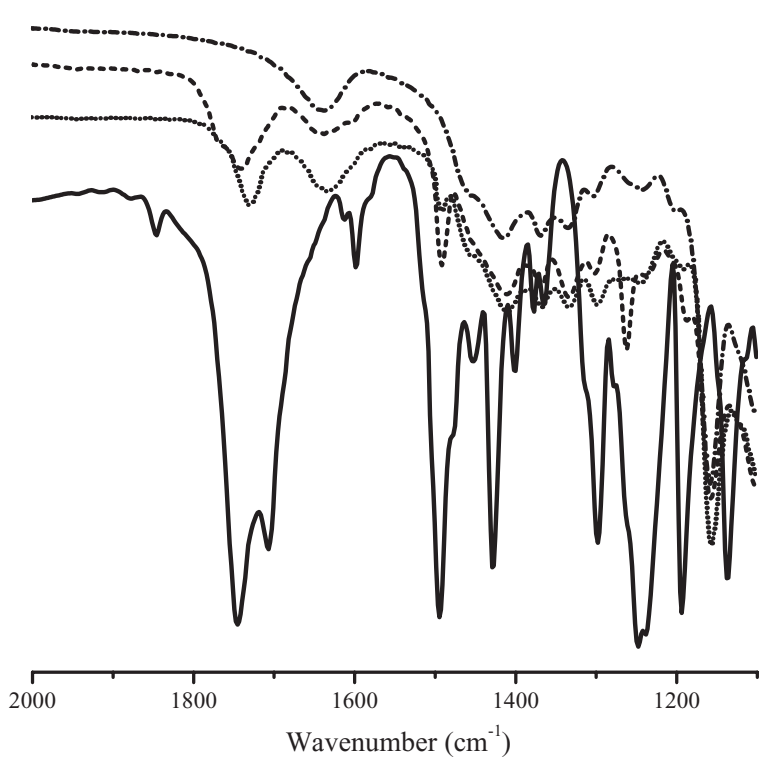

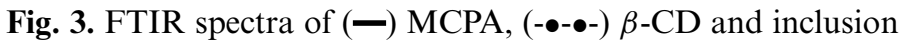

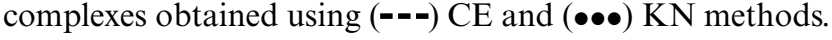




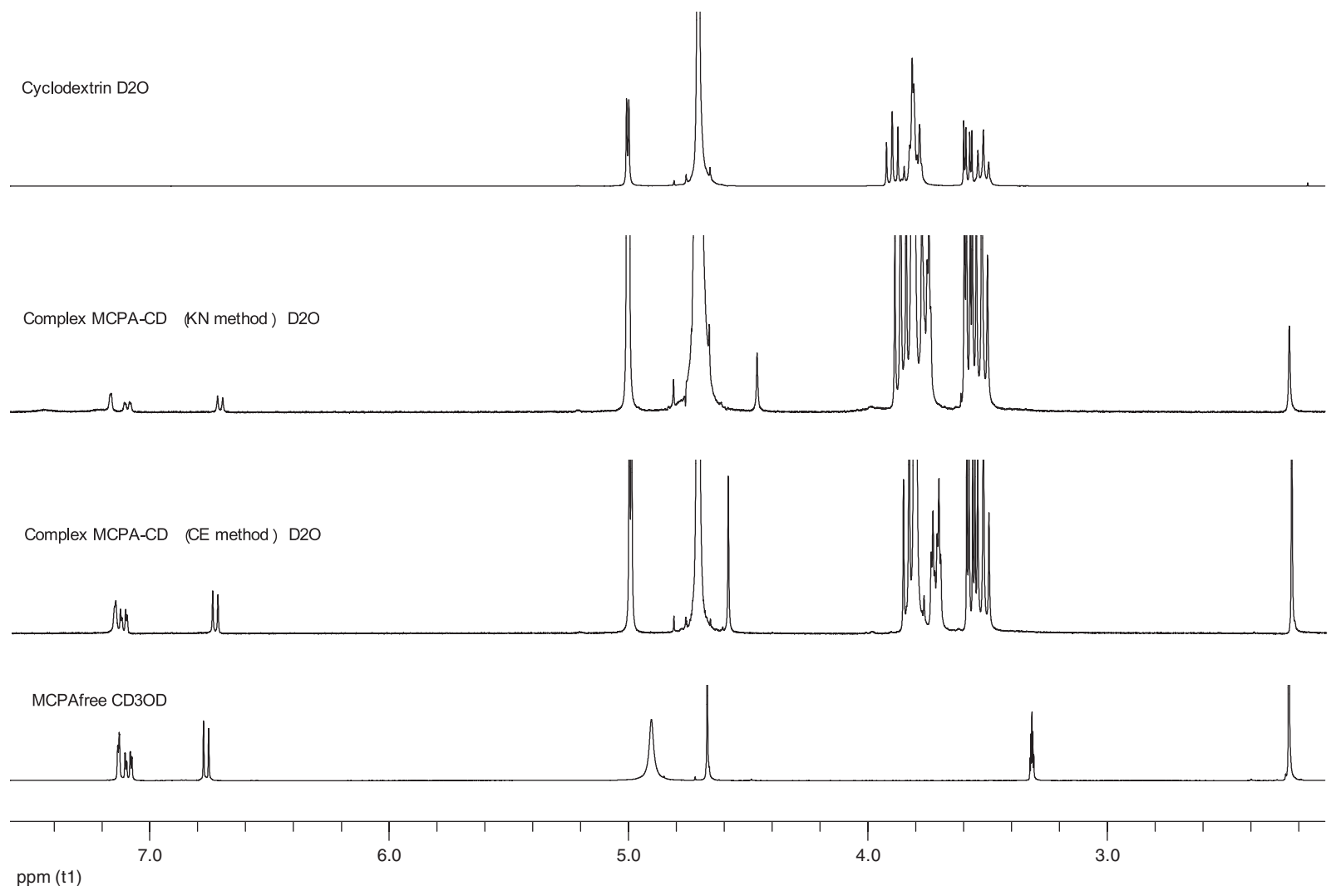

Fig. 4. ${ }^{1} \mathrm{H}$ NMR spectra of $\beta$-cyclodextrin $(\beta-\mathrm{CD}), \mathrm{MCPA}$ and MCPA/ $\beta$-CD complexes obtained using KN and CE methods.

The FTIR spectrum of MCPA (Fig. 3) shows a characteristic absorption band at $1744 \mathrm{~cm}^{-1}$ assigned to carboxylic $\mathrm{C}=\mathrm{O}$ stretching vibration $\left(v_{\mathrm{C}=\mathrm{O}}\right)$; three bands at 1597,1495 and $1450 \mathrm{~cm}^{-1}$, due to $\mathrm{C}=\mathrm{C}\left(v_{\mathrm{C}=\mathrm{C}}\right)$ of the aromatic ring; a band at $1427 \mathrm{~cm}^{-1}$ assigned to $\mathrm{CH}_{2}\left(\mathrm{OCH}_{2}\right.$ group $)$ scissoring vibration; ${ }^{[22]}$ a band at $1298 \mathrm{~cm}^{-1}$, corresponding to the $\mathrm{C}-\mathrm{O}\left(v_{\mathrm{C}-\mathrm{O}}\right)$ single bond stretching vibration; a wide band due to hydroxyl $\mathrm{O}-\mathrm{H}$ of the carboxylic acid group $\left(\delta_{\mathrm{O}-\mathrm{H}}\right)$ appears at $1245 \mathrm{~cm}^{-1}$.

In the physical mixture (PM), no variations on these wavenumber values were observed (data not shown). Nevertheless, the spectra of the inclusion complexes obtained by $\mathrm{CE}$ and $\mathrm{KN}$ methods showed the displacement of the band corresponding to $\mathrm{C}=\mathrm{O}$ stretching vibration $\left(v_{\mathrm{C}=\mathrm{O}}\right), 1738$ and $1728 \mathrm{~cm}^{-1}$ respectively, accompanied by a diminution of the band intensity. Moreover, the band at $1427 \mathrm{~cm}^{-1}$, assigned to $\mathrm{CH}_{2}\left(\mathrm{OCH}_{2}\right.$ group) scissoring vibrations present a significant diminution on its intensity and a shift towards lower wavenumbers (Fig. 3). The overall FTIR data highlight the formation of an inclusion complex in which MCPA is probably located inside of the ring of $\beta$-cyclodextrin, because significant intermolecular hydrogen bonding interactions were detected between the acetic acid side chain of the herbicide and the CD. In fact, the FTIR spectra reveal important changes in some vibrational modes as consequence of the host/guest interactions.
Nuclear magnetic resonance analysis (NMR). NMR is a powerful and simple technique that can be used in the study of the inclusion process of a guest in the hydrophobic cavity of $\beta$-CD. The formation of MCPA/ $\beta$-CD complex in solution can be confirmed by determining the chemical displacement of the proton signals of MCPA/ $\beta$-CD complexes with respect to the native samples per si (MCPA and $\beta$-CD) (Fig. 4). Table 2 presents the ${ }^{1} \mathrm{H}$ NMR chemical shift data $(\delta)$ of MCPA and $\beta$-CD before and after the formation of the inclusion complexes. The results show that, in general, significant chemical shift variations are observed. The proton signals are upfield shifted with respect to the native compounds indicating host-guest interactions. ${ }^{[23,24]}$

In cyclodextrins the $\mathrm{H} 3^{\prime}$ and $\mathrm{H} 5^{\prime}$ protons of the glucose units are facing the interior of the CD cavity whereas H6' protons are located at the rim with the primary alcohols. In turn, the $\mathrm{H} 2$ and $\mathrm{H} 4$ protons are at the opposite entrance of the cavity ${ }^{[25]}$ A detailed examination of the spectra of the MCPA/ $\beta$-CD complexes (Table 2 ) indicate that the experimental shifts of the $\beta$-CD cavity protons (H3' and H5') have a noticeable displacement. The observed modification of the protons present in the $\beta$-CD inner cavity can be justified by the complex formation with inclusion of MCPA and thus to the presence of ring current effects generated by the aromatic nucleus of MCPA that in the inclusion 
Table 2. ${ }^{1} \mathrm{H}$ NMR chemical shift $(\delta)$ data of MCPA, $\beta$-CD and MCPA/ $\beta$-CD complexes.
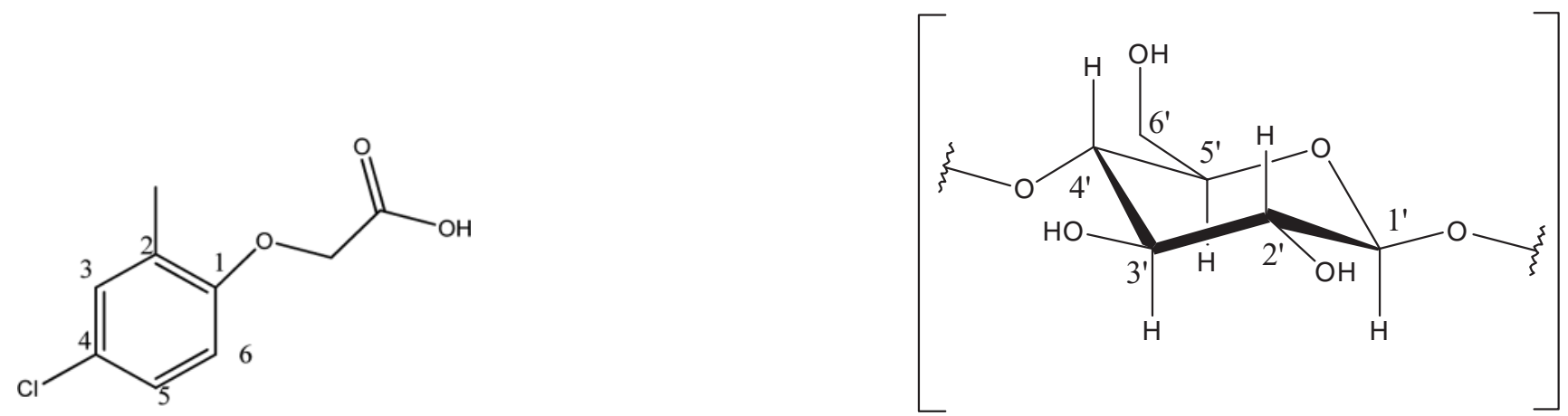

MCPA

$\beta-\mathrm{CD}$ unit

\begin{tabular}{|c|c|c|c|c|c|c|}
\hline $\begin{array}{l}H \\
\text { assignment } \delta\end{array}$ & $\begin{array}{c}M C P A \\
\left(C D_{3} O D\right)\end{array}$ & $\begin{array}{l}\text { cyclodextrin } \\
\left(\mathrm{D}_{2} \mathrm{O}\right)\end{array}$ & $\begin{array}{c}M C P A / \beta-C D \\
(K N \text { method }) \\
\left(\mathrm{D}_{2} \mathrm{O}\right)\end{array}$ & $\Delta \delta$ & $\begin{array}{c}M C P A / \beta-C D(C E \\
\text { method }\left(D_{2} \mathrm{O}\right)\end{array}$ & $\Delta \delta$ \\
\hline H3 & $7.129(\mathrm{~d})$ & - & 7.079 & -0.050 & 7.067 & -0.062 \\
\hline H5 & $7.087(\mathrm{dd})$ & - & 7.008 & -0.079 & 7.031 & -0.056 \\
\hline H6 & $6.761(\mathrm{~d})$ & - & 6.621 & -0.140 & 6.648 & -0.113 \\
\hline $\mathrm{OCH}_{2}$ & $4.666(\mathrm{~s})$ & - & 4.475 & -0.191 & 4.502 & -0.164 \\
\hline $\mathrm{CH}_{3}$ & $2.232(\mathrm{~s})$ & - & 2.149 & -0.083 & 2.146 & -0.086 \\
\hline $\mathrm{H} 1^{\prime}$ & - & $4.950(\mathrm{~d})$ & 4.915 & -0.035 & 4.912 & -0.038 \\
\hline $\mathrm{H} 3^{\prime}$ & & $3.843(\mathrm{t})$ & 3.775 & -0.068 & 3.746 & -0.097 \\
\hline $\mathrm{H} 6^{\prime}$ & - & $3.760(\mathrm{t})$ & 3.720 & -0.040 & 3.723 & -0.037 \\
\hline $\mathrm{H}^{\prime}$ & - & $3.734(\mathrm{~d})$ & 3.671 & -0.063 & 3.634 & -0.100 \\
\hline $\mathrm{H} 2^{\prime}$ & - & $3.527(\mathrm{~d})$ & 3.492 & -0.035 & 3.488 & -0.039 \\
\hline $\mathrm{H} 4^{\prime}$ & - & $3.462(\mathrm{t})$ & 3.435 & -0.027 & 3.436 & -0.026 \\
\hline
\end{tabular}

process affect the neighboring proton signals of the host (Fig. 4, Table 2).

In the ${ }^{1} \mathrm{H}$ NMR spectra of the MCPA/ $\beta$-CD complexes a marked effect ( $\delta$ complex $-\delta$ free) is also observed for $\mathrm{OCH}_{2}$ group and $\mathrm{H} 6$ aromatic proton (Table 2). This effect could be as well a consequence of the occurrence of a strong interaction between the host (MCPA) and the guest $(\beta-\mathrm{CD})$. This conclusion is in accordance with the data obtained by FTIR.

The guest position in the cyclodextrin is proposed in Figure 5. The proposal is consistent with the upfield shifts of the $\beta$-CD cavity protons as they experienced anisotropic shielding due to the inclusion of the MCPA aromatic ring.

The stoichiometry of complexes was also determined by comparing the ${ }^{1} \mathrm{H}$ NMR integration data (between the $\mathrm{H} 6$ proton in MCPA and H1' proton of the $\beta$-CD). ${ }^{[26]}$ For the MCPA/ $\beta$-CD complex obtained using the KN method, a relationship of approximately 1:14 was obtained that point out a stoichiometry 1:2 while for MCPA/ $\beta$-CD complex obtained using the CE method a relationship of 1:7 was obtained corresponding to a 1:1 stoichiometry. These results can be interpreted on the basis of the different techniques

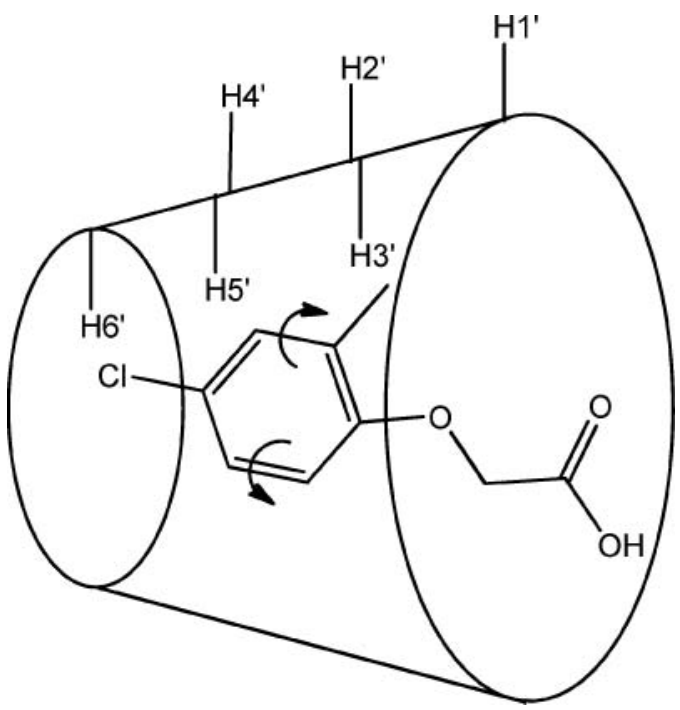

Fig. 5. Proposed structure for the $1: 1 \mathrm{MCPA} / \beta-\mathrm{CD}$ complex obtained using the CE method. 
used to prepare the CD complexes. The experimental results obtained for a $C D$ complex can depend on the technique used since the CD complexes are held together by weak forces. Moreover, the complexation process depends heavily on the experimental conditions $(\mathrm{pH}$, cosolvent, temperature, etc.). Thus, the complexes can involve species of different stoichiometries, depending on the technique used, in this case co-evaporation (CE) and kneading (KN) methods.

\section{Conclusion}

In recent years, $\beta$-CD has gained appreciable acceptance among the various types of cyclodextrins. The inclusion complexes of $\beta$-CD have been successfully used to improve solubility, chemical stability and bioavailability of a number of poorly soluble compounds. Accordingly, the inclusion complexation behavior, characterization and binding ability of the herbicide MCPA with $\beta$-CD were investigated. The combined use of different analytical techniques has provided a complete characterization of the MCPA/ $\beta$-CD inclusion complexes. The complexes obtained showed different structural and thermal properties and distinct spectroscopic features from the free molecules.

The gathered results allow concluding that $\beta$-CD can enhance the water solubility of MCPA, a fact that can be regarded as an important step in the design of novel MCPA formulations with enhanced chemical properties.

\section{Acknowledgments}

Financial support from Fundação para a Ciência e Tecnologia FCT/MCTES project PTDC/AGRAAM/105044/2008, National Funds PIDDAC also cofinanced by the European Community Fund FEDER through COMPETE-Programa Operacional Factores de Competitividade (POFC), is gratefully acknowledged.

\section{References}

[1] Gupta, P. K. Pesticide Exposure-Indian Scene. Toxicology 2004, 198, 83-90.

[2] Rodham, D. K. Colloid and interface science in formulation research for crop protection products. Curr. Opin. Colloid Interface Sci. 2000, 5, 280-287.

[3] Beestman, G. B. Controlled release in crop protection: past experience and future opportunities. In Chemistry of Crop Protection; Voss, G., Ramos, G., Eds.; Wiley: New York, 2003; 272 279.

[4] Sopeña, F.; Maqueda, C.; Morillo, E. Controlled release formulations of herbicides based on micro-encapsulation. Cien. Inv. Agr. 2009, 36, 27-42.

[5] Szejtli, J. Introduction and general overview of cyclodextrin chemistry. Chem. Rev. 1998, 98, 1743-1753.

[6] Martin Del Valle, E.M. Cyclodextrins and their uses: a review. Process Biochem. 2004, 39, 1033-1046.
[7] Villaverde, J. Time-dependent sorption of norflurazon in four different soils: Use of $\beta$-cyclodextrin solutions for remediation of pesticide-contaminated soils. J. Hazard. Mat. 2007, 142, 184 190.

[8] Zhang, A.; Liu, W. Inclusion effect of alpha-cyclodextrin on chemical degradation of malathionin water. Arch. Environ. Contam. Toxicol. 2008, 54, 355-362.

[9] Smith, V.J.; Bogdan, D.; Caira, M.R.; Bogdan, M.; Bourne, S.A.; Farcas, S.I. Cyclodextrin inclusion of four phenylurea herbicides: determination of complex stoichiometries and stability constants using solution ${ }^{1}$ H NMR spectroscopy. Supramol. Chem. 2010, 22, 172-177.

[10] Mortensen, S.K.; Jacobsen, C.S. Influence of frozen storage on herbicide degradation capacity in surface and subsurface sandy soils. Environ. Sci. Technol. 2004, 38, 6625-6632.

[11] Thorstensen, C.W.; Christiansen, A. Determination of bentazone, dichloroprop, and MCPA in different soils by sodium hydroxide extraction in combination with solid-phase preconcentration. J. Agric. Food Chem. 2001, 49, 4199-4202.

[12] Tomlin, C.D.S. The e-Pesticide Manual, 12th edition CD-ROM form, version 2.0; British Crop Protection Council, Hampshire, UK 2001.

[13] Eurostat. The use of plant protection products in the European Union: Data 1992-2003. Office for official publications of the European Communities: Luxembourg, 2007.

[14] van Ravenzwaay, B.; Pigott, G.; Leibold, E. Absorption, distribution, metabolism and excretion of 4-chloro-2-methylphenoxyacetic acid (MCPA) in rats. Food Chem. Toxicol. 2004, 42, 115-125.

[15] Bellet, E.M.; van Ravenzwaay, B.; Hellwig, J.; Pigott, G. Reproductive toxicity of MCPA (4-chloro-2-methylphenoxyacetic acid) in the rat. Int. J. Toxicol. 2001, 20, 29-38.

[16] Higuchi, T.; Connors, K.A. Phase-solubility techniques. Adv. Anal. Chem. Instrum. 1965, 4, 117-212.

[17] Topalov, A.; Abramovic, B.; Molnár-Gábor, D.; Csanádi, J.; Arcson, O. Photocatalytic oxidation of the herbicide (4-chloro-2methylphenoxy)acetic acid (MCPA) over $\mathrm{TiO}_{2}$. J. Photochem. Photobiol. A: Chem. 2001, 140, 249-253.

[18] Palmeiri, G.F.; Angeli, D.G.; Giovannnucci, G.; Martelli, S. Inclusion of methoxybutropate in $\beta$ - and hydroxylpropyl $\beta$-cyclodextrins: Comparison of preparation methods. Drug Dev. Ind. Pharm. 1997, 23, 27-37.

[19] Hedges, A.R. Industrial application of cyclodextrins. Chem. Rev. 1998, 98, 2035-2044.

[20] Challa, R.; Ahuja, A.; Ali, J.; Khar, R.K. Cyclodextrins in drug delivery: an updated review. AAPS PharmSciTech. 2005, 6, E329-E357.

[21] Gomez, M.J.; Bruneau, C.; Soyer, N.; Brault, A. Thermal degradation of chlorophenoxy acid herbicides. J. Agric. Food Chem. 1988, $36,649-653$.

[22] Sundaraganesan, N.; Meganathan, C.; Karthikeyan, B. FT-IR, FT-Raman spectra and quantum chemical calculations of some chloro substituted phenoxy acetic acids. Spectrochim. Acta A Mol. Biomol. Spectrosc. 2008, 70, 430-438.

[23] Ejchart, A.; Kozminski, W. NMR of cyclodextrins and their complexes. In Cyclodextrins and Their Complexes; Dodziuk, H., Ed.; Wiley-VCH: Weinheim, 2006; 231-254.

[24] Singh, R.; Bharti, N.; Madan, J.; Hiremath, S. N. Characterization of cyclodextrin inclusion complexes-a review. J.P.S.T. 2010, 2, 171-183.

[25] Jullian, C.; Morales-Montecinos, J.; Zapata-Torres, G.; Aguilera, B.; Rodriguez, J.; Arán, V.; Olea-Azar, C. NMR characterization, phase-solubility, and molecular modelling of inclusion complex of 5-nitroindazole derivative with cyclodextrins. Bioorg. Med. Chem. 2008, 16, 5078-5084.

[26] Chen, M.; Diao, G.; Zhang, E. Study of inclusion complex of beta-cyclodextrin and nitrobenzene. Chemosphere 2006, 63, 522529. 